\title{
Structure and Evolution of Confined Carbon Species during Methane Dehydroaromatization over Mo/ZSM-5
}

\author{
Nikolay Kosinov,*๑ Evgeny A. Uslamin, Ferdy J. A. G. Coumans, Alexandra S. G. Wijpkema, \\ Roderigh Y. Rohling, and Emiel J. M. Hensen*৫
}

Laboratory of Inorganic Materials Chemistry, Schuit Institute of Catalysis, Department of Chemical Engineering and Chemistry, Eindhoven University of Technology, P.O. Box 513, 5600 MB Eindhoven, The Netherlands

Supporting Information

ABSTRACT: Surface carbon (coke, carbonaceous deposits) is an integral aspect of methane dehydroaromatization catalyzed by Mo/zeolites. We investigated the evolution of surface carbon species from the beginning of the induction period until the complete catalyst deactivation by the pulse reaction technique, TGA, ${ }^{13} \mathrm{C}$ NMR, TEM, and XPS. Isotope labeling was performed to confirm the catalytic role of confined carbon species during MDA. It was found that "hard" and "soft" coke distinction is mainly related to the location
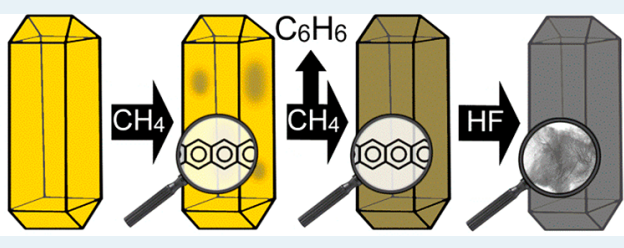
of coke species inside the pores and on the external surface, respectively. In addition, $\mathrm{MoO}_{3}$ species act as an active oxidation catalyst, reducing the combustion temperature of a certain fraction of coke. Furthermore, after dissolving the zeolite framework by HF, we found that coke formed during the MDA reaction inside the zeolite pores is essentially a zeolite-templated carbon material. The possibility of preparing zeolite-templated carbons from the most available hydrocarbon feedstock is important for the development of these interesting materials.

KEYWORDS: methane dehydroaromatization, Mo/ZSM-5, hydrocarbon pool, coke, zeolite-templated carbon

\section{INTRODUCTION}

Methane dehydroaromatization (MDA) is a promising technology for the direct conversion of methane, the main component of natural gas, to liquid aromatics. ${ }^{1}$ This reaction has not resulted in a commercial process yet, mainly because the benchmark MDA catalysts such as Mo/ZSM-5 and Mo/ MCM-22 quickly deactivate due to coking. Formation of coke over Mo/zeolite catalysts under the MDA conditions cannot be avoided because high temperatures $\left(\geq 700{ }^{\circ} \mathrm{C}\right)$ are needed for an appreciable conversion of methane to benzene. Under these conditions, polycyclic aromatic hydrocarbons and eventually graphite are more stable than the desired light aromatic products, requiring the use of shape-selective zeolites like ZSM-5 or MCM-22., ${ }^{2,3}$ Considering the relatively short lifetime of MDA catalysts, several approaches to decrease the coking rate have been investigated. For instance, a small amount of oxygen can be supplied to the reaction to continuously burn the coke species without extensive oxidation of the active Mo phase to Mo(VI). This can be achieved by using either oxygen-permeable ceramic membranes ${ }^{4-6}$ or short oxygen pulses. ${ }^{7}$ Complete regeneration of (partially) deactivated MDA catalysts through controlled reaction-regeneration cycling with hydrogen ${ }^{8,9}$ or oxygen ${ }^{10,11}$ has also been explored.

Despite significant progress in this area, the structure and evolution of the coke species deposited during the MDA reaction remain elusive. Temperature-programmed techniques such as temperature-programmed oxidation (TPO) and thermogravimetric analysis (TGA) are frequently used to characterize the carbonaceous deposits. ${ }^{12}$ TPO traces of coke formed during the MDA reaction typically contain several features attributed to molybdenum carbide, "soft" or molybdenum-associated coke, and "hard" coke associated with Brønsted acid sites. ${ }^{13-24}$ There are relatively few spectroscopic investigations of these coke deposits. Weckhuysen et al. performed an extensive XPS study to show that molybdenum carbide, $\mathrm{sp}^{2}$-type aromatic species and hydrogenpoor sp-type species dominate the external surface of spent Mo/ZSM-5. ${ }^{25}$ Tempelman et al. demonstrated by UV-Raman spectroscopy study that the coke species are polyaromatic hydrocarbons independent of time on stream. ${ }^{26}$

In a recent publication employing a pulse reaction technique and isotope labeling, we found that polyaromatic carbon species inside the zeolite pores are involved in the formation of benzene during the MDA reaction. ${ }^{27}$ We established that partially reduced isolated Mo-species catalyze the initial conversion of methane to yet unknown intermediates, which are involved in further reactions with the polyaromatic hydrocarbons eliminating benzene and other aromatics. The involvement of an organocatalytic intermediate resembles the hydrocarbon-pool mechanism for methanol-to-hydrocarbon (MTH) reactions. ${ }^{28}$ A pronounced difference is that the MDA reaction likely involves radicals, unlike the $\mathrm{MTH}$ reaction that proceeds via carbocation chemistry. Figure 1 provides a general scheme of the radical-based hydrocarbon pool mechanism for the MDA reaction.

Received: June 27, 2018

Revised: July 27, 2018

Published: August 15, 2018 


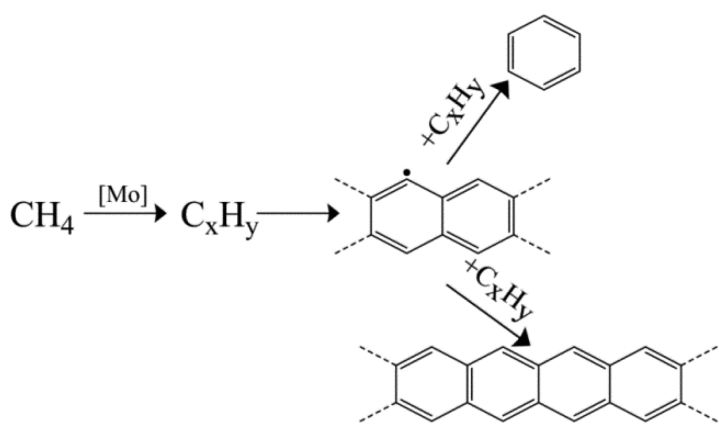

Figure 1. Scheme of the hydrocarbon pool MDA mechanism for Mo/ ZSM-5. The formation of linear polyaromatic intermediates takes place during the induction stage. Once the hydrocarbon pool is established the formation of light aromatics occurs in parallel with the further growth of polyaromatic species, eventually blocking the zeolite pores.

In this work, we focus on the carbonaceous deposits formed on Mo/ZSM-5 during the MDA reaction at $700{ }^{\circ} \mathrm{C}$ using multiple characterization techniques. First, we follow the rapid accumulation of carbon in the micropores of ZSM-5 during the induction period. Isotope labeling with ${ }^{13} \mathrm{C}$ and $\mathrm{D}$ is employed to further underpin the conclusion that confined coke species are actively involved in the MDA reaction. Second, we analyze the evolution of coke species during prolonged MDA reaction by ${ }^{13} \mathrm{C}$ MAS NMR and DTG and demonstrate the limited ability of TPO-based techniques for studying the chemical speciation of coke. Finally, we characterize the carbon material in spent MDA catalysts after removal of the zeolite by $\mathrm{HF}$ etching.

\section{EXPERIMENTAL SECTION}

Preparation of Materials. Supported Mo catalysts were prepared by incipient wetness impregnation of HZSM-5 powder (Si/Al 13, Süd-Chemie, now Clariant) with an aqueous solution of ammonium heptamolybdate tetrahydrate $\left(\mathrm{NH}_{4}\right)_{6} \mathrm{Mo}_{7} \mathrm{O}_{24} \cdot 4 \mathrm{H}_{2} \mathrm{O}$ (Merck) of appropriate concentration. After impregnation, the catalysts were dried at $110{ }^{\circ} \mathrm{C}$ overnight and calcined in air at $550{ }^{\circ} \mathrm{C}$ for $6 \mathrm{~h}$ with a ramp rate of $2{ }^{\circ} \mathrm{C} / \mathrm{min}$. Depending on Mo loading, the catalysts were denoted as $1 \%$ Mo (1 wt \% of Mo metal loading), $2 \%$ Mo, and $5 \%$ Mo. Additionally, Mo-containing samples supported on activated carbon (RX-3, Norit), silicalite-1 (prepared according to ref 29), and silica (Grace 643) were prepared by the same method and denoted as Mo/AC (this sample was not calcined at $550{ }^{\circ} \mathrm{C}$ ), Mo/silicalite- 1 , and $\mathrm{Mo} /$ silica, respectively.

To liberate the carbonaceous deposits from the zeolite, an amount of $1 \%$ Mo spent sample was dissolved in an excess of HF (40\%) for $2 \mathrm{~h}$. The resulting solid was washed with a copious amount of water on a paper filter and finally dried at $110^{\circ} \mathrm{C}$ overnight.

Catalyst Testing and Characterization. The prepared catalysts were pelletized, crushed, and sieved to obtain particles with a size of $0.25-0.5 \mathrm{~mm}$. An amount of $0.3 \mathrm{~g}$ of sieved catalyst was placed into a tubular quartz reactor (i.d. $4.0 \mathrm{~mm}$, length $250 \mathrm{~mm}$ ). The catalyst was supported by quartz wool plugs inside the isothermal $\left(700{ }^{\circ} \mathrm{C}\right)$ zone of the reactor. Typically, a $15 \mathrm{~mL} / \mathrm{min}$ flow of $\mathrm{CH}_{4} / \mathrm{N}_{2}$ mixture (95:5) was used, which represents a $\mathrm{CH}_{4}$ WHSV of $2.0 \mathrm{~h}^{-1}$. For the isotope-labeling studies, ${ }^{13} \mathrm{CH}_{4}\left(99 \%{ }^{13} \mathrm{C}\right.$, Euriso-Top) and $\mathrm{CD}_{4}$ (99\% D, Euriso-Top) were used.

${ }^{13} \mathrm{C}$ NMR MAS NMR spectra of coked catalysts were recorded on a $4.7 \mathrm{~T}$ Bruker DRX-200 NMR spectrometer operating at $50.3 \mathrm{MHz}$. The measurements were performed using a $4 \mathrm{~mm}$ MAS probe head with a sample rotation rate of 8-10 kHz. Quantitative spectra were recorded using the highpower proton decoupling direct excitation (HPDEC) pulse sequence with an interscan delay of $10 \mathrm{~s}$. The spectra were referenced to the ${ }^{13} \mathrm{C}$ signal of adamantane at $38.56 \mathrm{ppm}$. X-ray diffraction (XRD) was performed with a Bruker D2 powder diffraction system $\left(\mathrm{Cu} \mathrm{K} \alpha\right.$ radiation, scan speed $0.01^{\circ} / \mathrm{s}, 2 \theta$ range 5 to $40^{\circ}$ ). The external surface concentration of Mo species was estimated by X-ray photoelectron spectroscopy (XPS) with a Thermo Scientific K- $\alpha$ spectrometer equipped with a monochromatic $\mathrm{Al} \mathrm{K} \alpha(1486.6 \mathrm{eV}) \mathrm{X}$-ray source and a $180^{\circ}$ double-focusing hemispherical analyzer with a 128channel detector. Textural properties were analyzed by $\mathrm{Ar}$ physisorption carried out at $-186{ }^{\circ} \mathrm{C}$ using a Micromeritics ASAP-2020 apparatus. Nonlocal density functional theory (NLDFT) was used to determine the pore size distribution of the materials. Prior to the measurements, the samples were outgassed at $400{ }^{\circ} \mathrm{C}$ overnight. For the thermogravimetric analysis (TGA) of coke species, a Mettler Toledo TGA/DSC 1 instrument was applied. An amount of ca. $25 \mathrm{mg}$ of spent catalyst was placed in an uncovered alumina crucible, which was heated to $900{ }^{\circ} \mathrm{C}$ at a rate of $5{ }^{\circ} \mathrm{C} / \mathrm{min}$ in a $40 \mathrm{~mL} / \mathrm{min}$

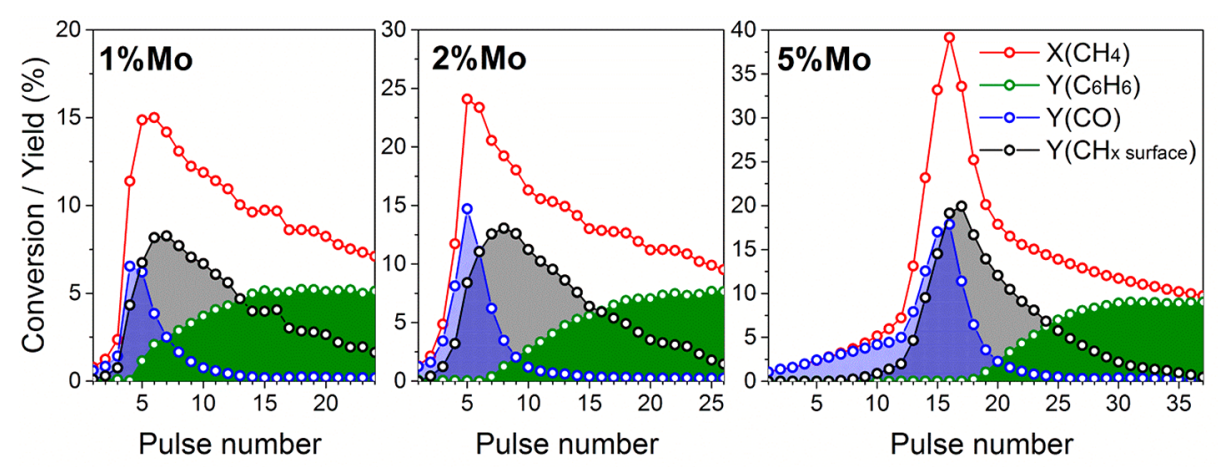

Figure 2. Per-pulse conversion of methane and yields of surface carbon species (estimated by the carbon balance) and benzene during pulsing methane over Mo/ZSM-5 catalysts. Stages of Mo(VI) reduction (activation); extensive formation of surface carbon (induction) and quasi-steadystate benzene production can be distinguished. Additionally Figure S1 shows catalytic performance under flow conditions, and Figure S2 presents the distribution of all detected products during the pulse reaction test. Conditions: $700{ }^{\circ} \mathrm{C}, 5 \mathrm{~mL}$ methane pulses every $200 \mathrm{~s}, 30 \mathrm{~mL} / \mathrm{min}$ flow of Ar carrier, MS analysis. 

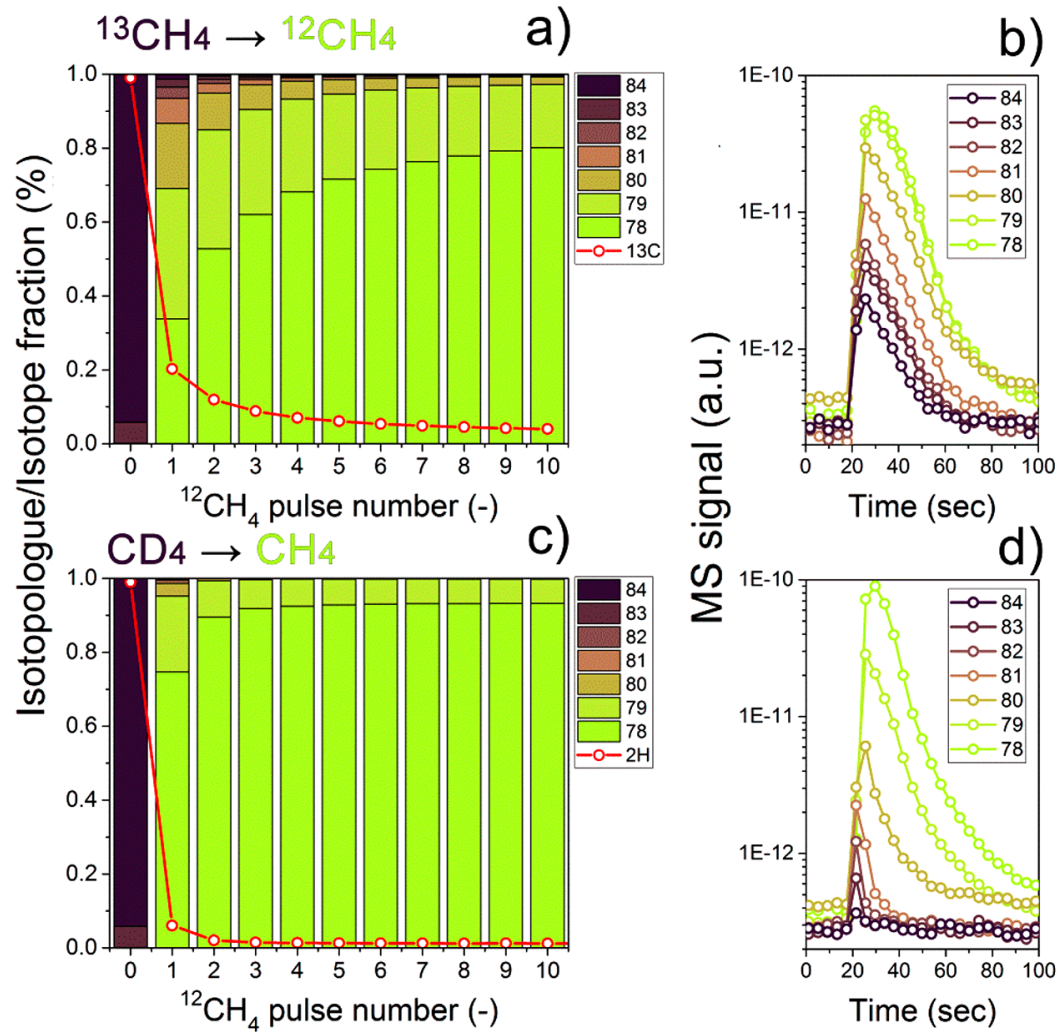

Figure 3. Pulsing isotope exchange results with ${ }^{13} \mathrm{CH}_{4} \rightarrow{ }^{12} \mathrm{CH}_{4}$ (a, b) and $\mathrm{CD}_{4} \rightarrow \mathrm{CH}_{4}$ (c, d) switching over the $2 \%$ Mo catalyst, which was induced by 14 pulses of ${ }^{13} \mathrm{CH}_{4}$ and 20 pulses of $\mathrm{CD}_{4}$, followed by a thorough flushing with Ar flow and pulsing nonlabeled methane. Distribution of benzene isotopologues just before switching to unlabeled methane and for the next 10 pulses after the switch (a, $c$ ), MS profiles of benzene signals during the first pulse after the switch (b, d). Conditions: $700{ }^{\circ} \mathrm{C}, 0.3 \mathrm{~g}$ of $2 \%$ Mo catalyst, $5 \mathrm{~mL}$ pulses every $200 \mathrm{~s}, \mathrm{Ar}$ carrier flow $30 \mathrm{~mL} / \mathrm{min}$.

$\mathrm{He}+20 \mathrm{~mL} / \mathrm{min} \mathrm{O}_{2}$ flow. The morphology of the zeolite and carbon particles was analyzed by scanning electron microscopy (SEM) with a FEI Quanta 200F scanning electron microscope at an accelerating voltage of $3-5 \mathrm{kV}$ using aluminum sample holders. Transmission electron microscopy (TEM) micrographs were obtained with a FEI Tecnai 20 instrument at an electron acceleration voltage of $200 \mathrm{kV}$.

Modeling of Carbon Species. A structural model of MFItemplated carbon was constructed in Materials Studio 6.0 (Biovia, formerly Accelrys Software, Inc.). The zeolite structure of MFI (silicalite-1) was taken from the internal database. The polyaromatic structure confined inside the silicalite-1 pores was subjected to a geometry optimization using the COMPASS force field at energy and force convergence criteria of 0.1 and $0.5 \mathrm{cal} / \mathrm{mol} / \AA$, respectively. The Ewald summation method was employed to account for the electrostatic and van der Waals contributions to the energy. The zeolite framework was removed after this step in order to analyze the pore sizes of the resulting carbon material.

\section{RESULTS AND DISCUSSION}

Formation of Carbon Species during the Induction Period. We followed the initial stage of the MDA reaction by supplying short pulses of methane to the Mo/ZSM-5 catalyst bed as described before. ${ }^{27}$

As Figure 2 shows, we can distinguish three reaction stages: (i) activation, involving the reduction of $\mathrm{Mo}(\mathrm{VI})$ with $\mathrm{CO}$ as main reaction product; (ii) induction, involving formation of a significant amount of surface carbon, which can be linked to an increasing amount of benzene formation; and (iii) quasi- steady-state production of benzene (and other light aromatics). The results show that the formation of surface polyaromatics precedes that of benzene independent of the Mo loading. This sequence of reactions can be explained by the following scheme:

$$
\mathrm{CH}_{4} \rightarrow \mathrm{CH}_{x \text { surface }} \rightarrow \mathrm{C}_{6} \mathrm{H}_{6}
$$

Further, we used isotope labeling to determine the involvement of surface carbon and proton atoms into the produced aromatic molecules. These experiments were performed by activating and inducing the catalysts with ${ }^{13} \mathrm{CH}_{4}$ or $\mathrm{CD}_{4}$, followed by thorough flushing with $\mathrm{Ar}$ to remove any gaseous organic molecules from the system (until complete disappearance of organic MS signals). Finally, pulses of unlabeled $\mathrm{CH}_{4}$ were supplied to the catalyst bed. Figure 3 shows that the surface carbon and hydrogen atoms are incorporated into the benzene molecules. After activation and induction by ${ }^{13} \mathrm{CH}_{4}$ pulses, more than $65 \%$ of the produced benzene molecules contain at least one ${ }^{13} \mathrm{C}$ atom during the first ${ }^{12} \mathrm{CH}_{4}$ pulse. The total ${ }^{13} \mathrm{C}$ concentration in benzene is $20.3 \%$. Upon further ${ }^{12} \mathrm{CH}_{4}$ pulsing, the concentration of ${ }^{13} \mathrm{C}$ labels in the benzene molecules expectedly decreases. However, even after $10{ }^{12} \mathrm{CH}_{4}$ pulses the ${ }^{13} \mathrm{C}$ concentration is about three times higher than the natural ${ }^{13} \mathrm{C}$ abundance (1.1\%). Although D incorporation was less pronounced than ${ }^{13} \mathrm{C}$ incorporation, the $\mathrm{D}$ content also gradually decreased when more $\mathrm{CH}_{4}$ was pulsed. Apparent low degree of $\mathrm{D}$ incorporation can be explained by a low $\mathrm{H} / \mathrm{C}$ ratio of confined hydrocarbons and fast H/D exchange of both methane and benzene molecules under applied conditions. 
The distribution of benzene isotopologues obtained from the ${ }^{13} \mathrm{C}$ isotope experiments (Figure $4 \mathrm{a}$ ) is close to the
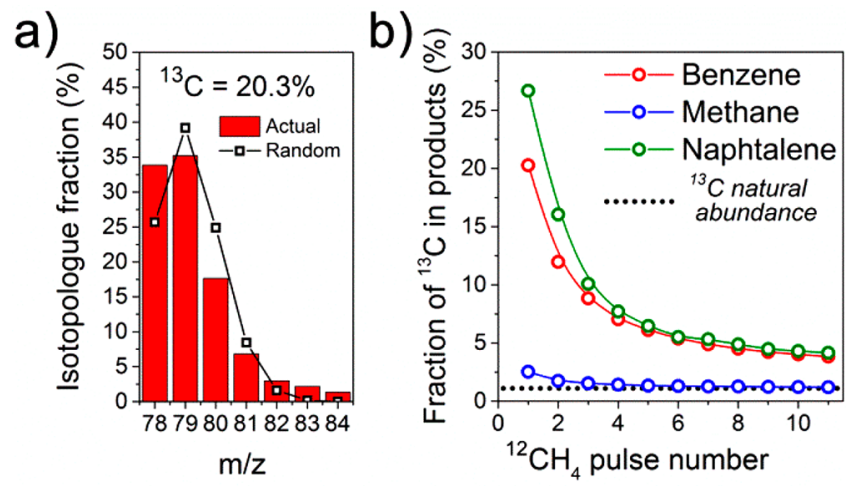

Figure 4. (a) Distribution of benzene isotopologues after the first ${ }^{12} \mathrm{CH}_{4}$ pulse plotted together with the random distribution corresponding to $20.3 \%$ concentration of ${ }^{13} \mathrm{C}$ atoms. (b) Development of ${ }^{13} \mathrm{C}$ incorporation into methane, benzene, and naphthalene molecules with the number of ${ }^{12} \mathrm{CH}_{4}$ pulses after the switch. The dashed line represents the $1.1 \%$ natural abundance of ${ }^{13} \mathrm{C}$.

distribution expected from a random statistical distribution at $20.3 \%$ of ${ }^{13} \mathrm{C}$. A slight excess of heavy isotopologues ${ }^{13} \mathrm{C}_{6} \mathrm{H}_{6}$, ${ }^{13} \mathrm{C}_{5}{ }^{12} \mathrm{C}_{1} \mathrm{H}_{6}$, and ${ }^{13} \mathrm{C}_{4}{ }^{12} \mathrm{C}_{2} \mathrm{H}_{6}$ and pure ${ }^{12} \mathrm{C}_{6} \mathrm{H}_{6}$ was observed. This observation implies that formed benzene might be in equilibrium with surface carbon species. On the other hand, the concentration of labeled carbon in methane molecules is much lower than that in benzene and naphthalene molecules (Figure $4 \mathrm{~b}$ ). Therefore, the observed isotope exchange is not the result of coke hydrogenation to form labeled methane, which is then further converted to labeled benzene. In other words the following reaction sequence does not significantly contribute to the process:

$$
\begin{aligned}
& { }^{13} \mathrm{CH}_{x \text { surface }}+\mathrm{H}_{2} \rightarrow{ }^{13} \mathrm{CH}_{4} \\
& { }^{12} \mathrm{CH}_{4}+{ }^{13} \mathrm{CH}_{4} \rightarrow{ }^{12} \mathrm{C}_{k}{ }^{13} \mathrm{C}_{6-k} \mathrm{H}_{6}+\mathrm{H}_{2}
\end{aligned}
$$

In such a case, the concentration of ${ }^{13} \mathrm{C}$ label in methane and in aromatic molecules would have to be the same. As the ${ }^{13} \mathrm{C}$ labels are included selectively into the aromatic molecules, the following overall reaction takes place:

$$
{ }^{12} \mathrm{CH}_{4}+{ }^{13} \mathrm{CH}_{x \text { surface }} \rightarrow{ }^{12} \mathrm{C}_{k}{ }^{13} \mathrm{C}_{6-k} \mathrm{H}_{6}+\mathrm{H}_{2}
$$

In this mechanism, mixed benzene isotopologues stem from initial methane activation products (e.g., methyl radicals or ethylene) reacting with the surface species. Previously, we reported that a part of the confined polyaromatic species has a radical character. We also found a correlation between the concentration of organic radicals and the amount of benzene formed during the induction stage. ${ }^{27}$ Therefore, we argue that radical pathways likely play an important role in the formation of benzene via surface intermediates.

Pulse reactions and isotope labeling studies showed that the surface carbon formed in the Mo/ZSM-5 pores in addition to the deactivation plays an active intermediate role in the MDA catalytic cycle. The insertion of surface carbon and hydrogen atoms into the benzene molecules likely proceeds via a reaction between the confined polyaromatics and the products of initial methane activation. Under the applied conditions, the contribution of scrambling through reverse hydrogenation of benzene and surface carbon to methane is not significant.

Evolution of Surface Carbon Species. The results presented in the previous section are related to the induction period, i.e., the very beginning of the MDA process. In the following sections, we discuss the properties of carbon species formed during the later reaction stages. First, we determined the remaining microporous volume of Mo/ZSM-5 catalysts by Ar physisorption and the total coke content by TG analysis as functions of the time on stream of the MDA reaction. The linear correlation between the amount of coke and the remaining microporous volume (Figure 5) suggests that the

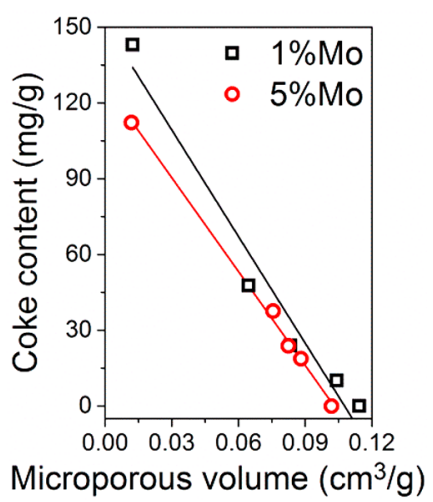

Figure 5. Correlation of the remaining microporous volumes of $1 \%$ Mo and 5\% Mo catalysts with the coke content. The samples were produced by performing the MDA reaction at $700{ }^{\circ} \mathrm{C}$ for $0,5,15,60$, and $960 \mathrm{~min}$.

carbon is predominantly located on the internal surface. As shown by Okkel et al., ${ }^{30}$ the deposition of carbon on the external surface of the zeolite would not result in a significant decrease of microporosity that can be probed by $\mathrm{Ar}$ physisorption.

Ar physisorption results demonstrate that carbon species block the pores of $1 \%$ Mo and 5\% Mo catalysts in a similar manner, independent of the Mo content. In contrast, the results of TG analysis are quite dependent on the Mo loading. Figure 6 shows that increasing the Mo content leads to an increased contribution of "soft" coke at about $450-550{ }^{\circ} \mathrm{C}$. Furthermore, the main combustion feature at $550-600{ }^{\circ} \mathrm{C}$ shifts to a lower temperature as the Mo loading increases. In
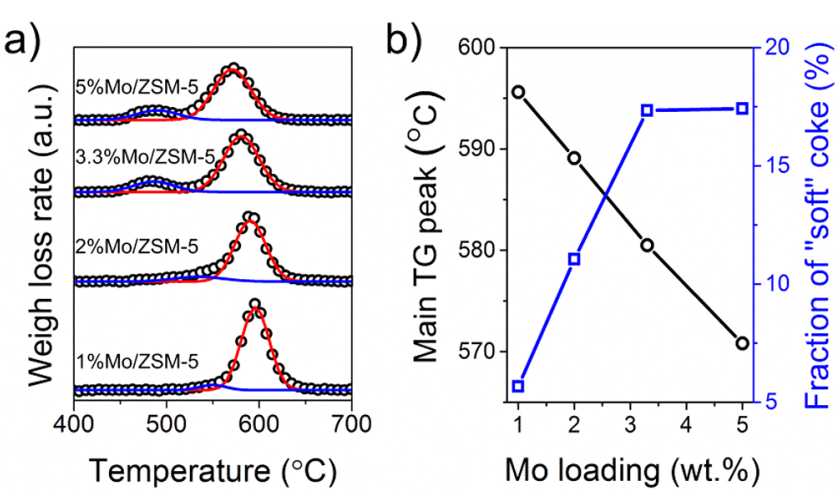

Figure 6. (a) DTG profiles and (b) dependences of the temperature of the main TG peak and the concentration of "soft" coke on Mo loading for Mo/ZSM-5 catalysts exposed to methane at $700{ }^{\circ} \mathrm{C}$ for 16 h. 
our previous work we showed that at a Mo loading above $2 \mathrm{wt}$ $\%$ a significant fraction of Mo remains as large Mo-oxo clusters on the external surface of fresh catalysts. ${ }^{11}$

XPS results (Figure 7) demonstrate that the concentration of Mo on the external surface of 5\% Mo catalyst is much higher

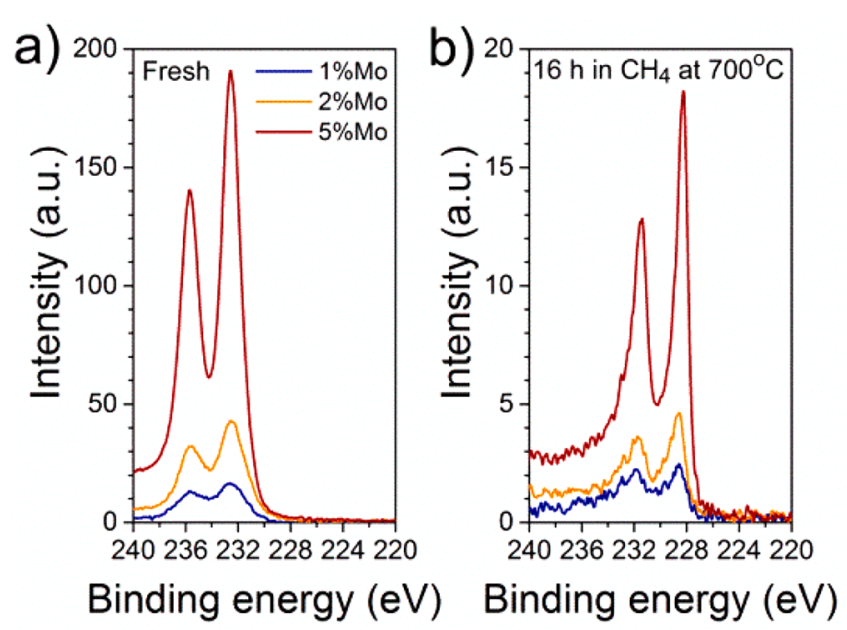

Figure 7. Mo 3d XPS spectra of $1 \%$ Mo/ZSM-5, 2\% Mo/ZSM-5, and $5 \%$ Mo/ZSM- 5 catalysts (a) before and (b) after the reaction under methane flow at $700{ }^{\circ} \mathrm{C}$ for $16 \mathrm{~h}$.

than on $2 \% \mathrm{Mo} / \mathrm{ZSM}-5$ and particularly $1 \% \mathrm{Mo} / \mathrm{ZSM}-5$ catalysts, not only before but also after the MDA reaction. Reduced Mo clusters on the external surface are known to be easily oxidized to $\mathrm{MoO}_{3},{ }^{7}$ which can catalyze the combustion of coke and therefore be responsible for the low-temperature DTG feature. Indeed, impregnation of activated carbon (AC) with increasing amounts of ammonium heptamolybdate gradually decreases the temperature necessary to oxidize the carbon from $600{ }^{\circ} \mathrm{C}$ (for pristine AC sample) to $450{ }^{\circ} \mathrm{C}$ for the $5 \% \mathrm{Mo} / \mathrm{AC}$ sample (Figure 8 ). Clearly, $\mathrm{MoO}_{3}$ is an efficient

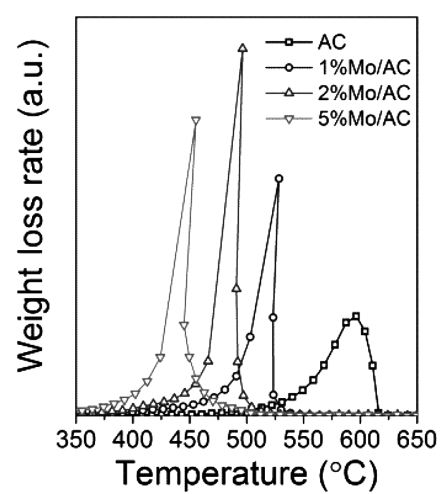

Figure 8. DTG profiles of activated carbon and the same carbon impregnated with 1,2 , and 5 wt \% Mo metal present in the form of ammonium heptamolybdate.

combustion catalyst that, if present on the external zeolite surface, can influence the combustion behavior of carbonaceous materials. We argue therefore that the apparent high amount of soft coke on $5 \% \mathrm{Mo} / \mathrm{ZSM}-5$ is most likely related to the $\mathrm{MoO}_{3}$-catalyzed combustion of carbon species located on/ near the external zeolite surface. Moreover, the heat generated during the combustion of these species results in the shift of the main DTG to lower temperature.
As the majority of carbon species are located in the micropores, XPS cannot be used to characterize these species (Figure S3). To examine the chemical nature and evolution of coke species we performed a ${ }^{13} \mathrm{C}$ NMR spectroscopy study, applying labeled ${ }^{13} \mathrm{CH}_{4}$ for the samples with low carbon content to enhance the quality of spectra. First, we analyzed $5 \%$ Mo catalysts exposed to methane for different periods of time and found that the DTG-derived amount of "hard" coke gradually increases with time on stream at the expense of the "soft" coke contribution (Figure 9a). This observation can be explained by a slow diffusion of oxygen and combustion products inside the micropores at increasing carbon content. ${ }^{31}$ The NMR spectra of these samples feature only one NMR signal at about $130 \mathrm{ppm}$ (Figure 9b). The intensity of the signal increases with time on stream but the position and relative width remain the same. This signal is assigned to $\mathrm{sp}^{2}$ carbon of aromatic nature. No signals corresponding to $\mathrm{sp}$ or $\mathrm{sp}^{3}$ carbon were observed.

A comparison of NMR and DTG data shows that the combustion properties of coke are not related to its structure, as samples with completely different "hard/soft" coke distribution are very similar from the NMR perspective. To underpin this conclusion, we also analyzed spent nonacidic $\mathrm{Mo} /$ silicalite- 1 and nonmicroporous $\mathrm{Mo} / \mathrm{SiO}_{2}$ samples. Spent $\mathrm{Mo} / \mathrm{SiO}_{2}$ is characterized by a single low-temperature DTG feature, while Mo/silicalite-1 displays a low- and a hightemperature feature similar to Mo/MFI sample but shifted to lower temperatures (Figure 9c). A stronger contribution of "soft" coke in Mo/silicalite-1 can be explained by the higher external surface concentration of $\mathrm{Mo}$ on the $\mathrm{SiO}_{2}$ support that does not contain Brønsted acid sites. ${ }^{32}$ Despite these differences, ${ }^{13} \mathrm{C}$ NMR spectra of spent Mo/silicalite-1 and Mo/ZSM-5 are similar (Figure 9d). The ${ }^{13} \mathrm{C}$ NMR signal in spent $\mathrm{Mo} / \mathrm{SiO}_{2}$ is significantly broadened, characteristic of large (pre)graphitic structures, formed on the surface of silica in absence of micropores. ${ }^{33}$

To summarize, the ${ }^{13} \mathrm{C}$ NMR and DTG data show that there is no significant chemical difference between the MDA coke burning at low temperature and at high temperature. The lowtemperature contribution is related to $\mathrm{MoC}_{x}$ species located on the external surface of spent samples. These species are easily oxidized to $\mathrm{MoO}_{3}$, which is an efficient combustion catalyst. The high-temperature contribution is related to the decreased accessibility of oxygen to the zeolite micropores. The transport of these molecules in the zeolite pores decreases with increasing coke content, resulting in the shift of the combustion features to the higher temperatures and the apparent "hardening" of the coke species.

Liberation of Carbon Material from the Zeolite. In order to gain a deeper insight into the properties of carbonaceous deposits formed during MDA, we dissolved a spent $\left(16 \mathrm{~h}\right.$ in methane flow at $\left.700{ }^{\circ} \mathrm{C}\right) 1 \%$ Mo catalyst in $\mathrm{HF}$ solution, filtered the remaining solid off, and thoroughly washed it with water. The ash content of the obtained carbon product was below $4 \%$ (Figure 10, inset). After liberation of the coke from the zeolite pores, its combustion profile shifted $\left(\sim 100{ }^{\circ} \mathrm{C}\right)$ to a significantly lower temperature (Figure 10$)$. This finding is in line with the conclusion that burning the coke molecules inside the zeolite pores is more difficult due to the presence of coke that blocks diffusion of oxygen.

Electron microscopy analysis reveals that the liberated carbonaceous deposits remain particulate and the size and shape of the primary particles are comparable to the parent 

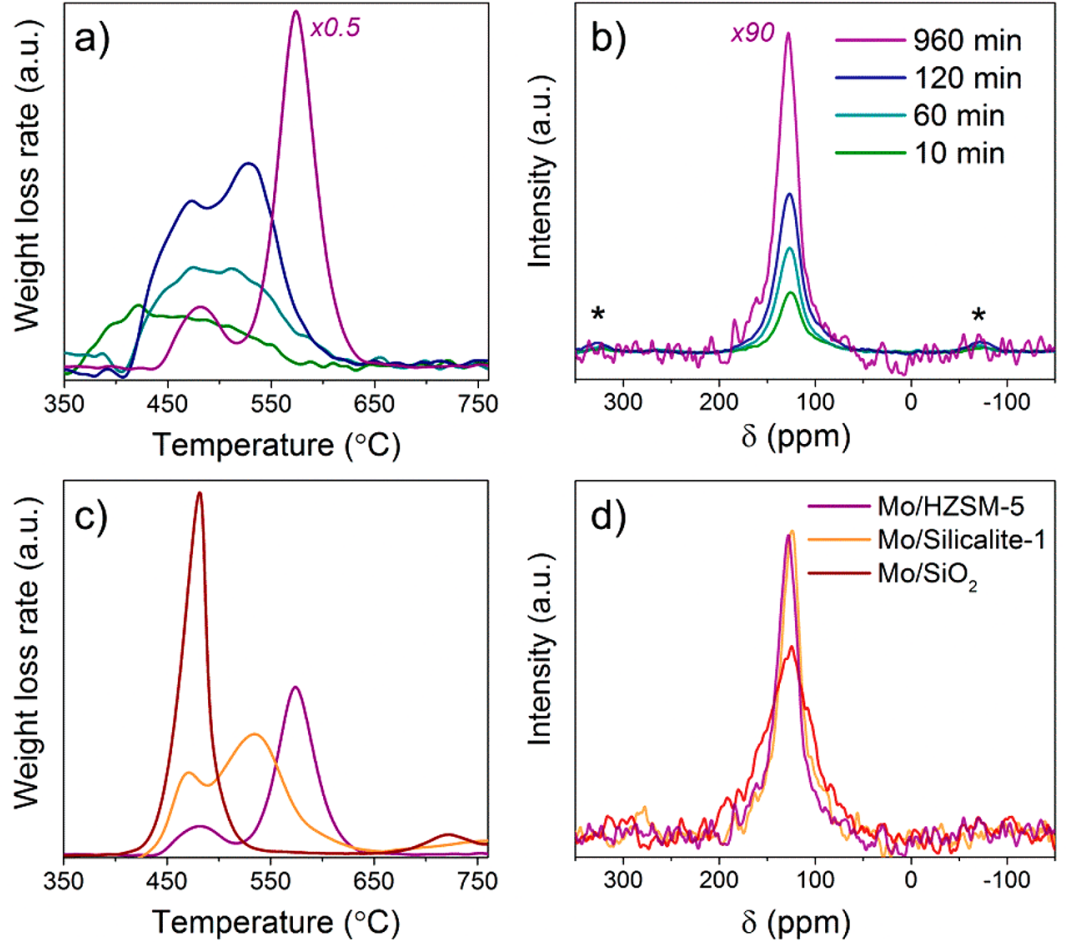

Figure 9. DTG profiles (a, c) and ${ }^{13} \mathrm{C}$ MAS NMR spectra (b, d) of $5 \% \mathrm{Mo} / \mathrm{ZSM}-5$ samples exposed to methane at $700{ }^{\circ} \mathrm{C}$ for different times (a, b) and comparison with $5 \% \mathrm{Mo} /$ silicalite- 1 and $10 \% \mathrm{Mo} /$ silica catalysts after $16 \mathrm{~h}$ of $\mathrm{CH}_{4}$ treatment. Labeled ${ }^{13} \mathrm{CH}_{4}$ methane was used to prepare the $5 \%$ Mo samples, except of 5\%, 960 min. Spent $\mathrm{Mo} /$ silicalite-1 and $\mathrm{Mo} / \mathrm{SiO}_{2}$ samples were prepared with normal $\mathrm{CH}_{4}$.

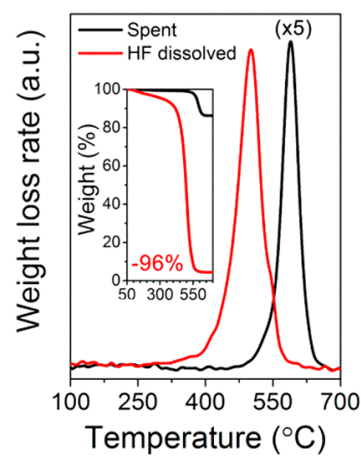

Figure 10. DTG profiles of $1 \%$ Mo-spent (black) and the product remained after dissolving the inorganic fraction in $\mathrm{HF}$ (red). Inset shows TG profiles of the two samples; inorganic fraction of the sample after dissolution in HF is below $4 \%$.

zeolite crystals (Figure 11). Moreover, since we did not observe any hollowness of the particles by TEM (Figure 11c), it can be concluded that the polyaromatic species (inter)grow throughout the whole zeolite crystal volume.
The analysis of the Ar adsorption isotherm of the liberated carbon material (Figure 12) shows that it possesses a
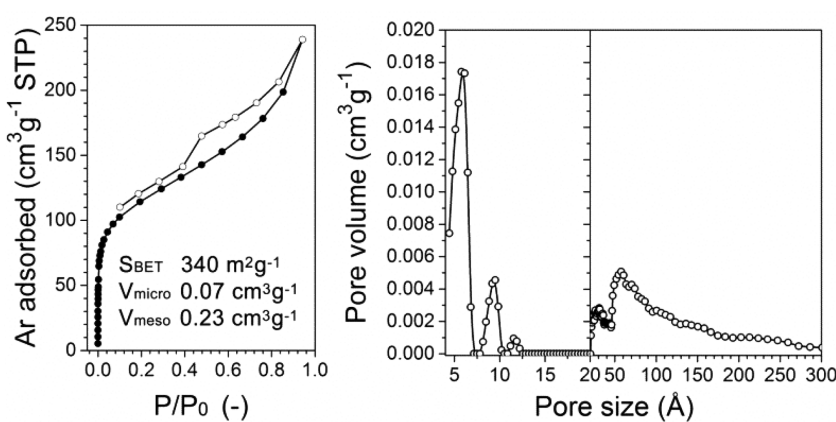

Figure 12. Ar adsorption isotherm (left) and NLDFT-derived pore size distribution (right) of $1 \%$ Mo-spent sample after dissolving the inorganic fraction in HF.

significant microporous volume of $0.07 \mathrm{~cm}^{3} / \mathrm{g}$ with micropore distribution peaks centered around 5.9, 9.0, and 12.0 A. The
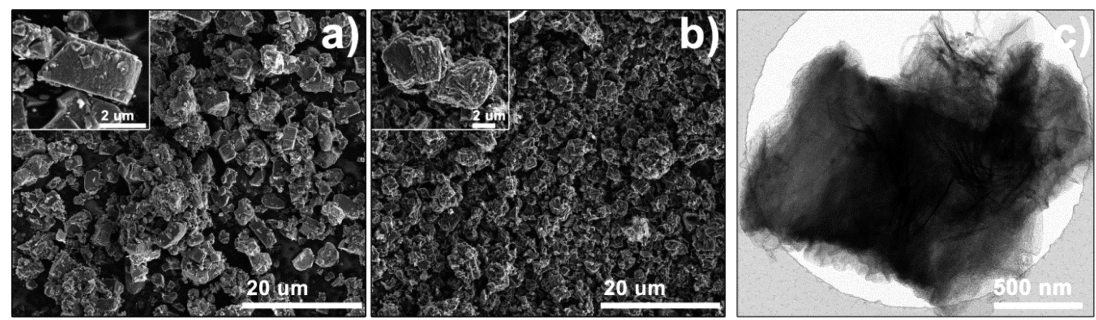

Figure 11. SEM image of (a) 1\% Mo-spent sample, (b) SEM and (c) TEM images of the carbon product remained after dissolving the inorganic fraction of $1 \%$ Mo-spent in HF. 
carbon material also contains mesopores (mesoporous volume of $0.23 \mathrm{~cm}^{3} / \mathrm{g}$ ) and the total BET surface area is $340 \mathrm{~m}^{2} / \mathrm{g}$.

The properties of the carbonaceous deposits formed during MDA are similar to those of zeolite-templated carbons (ZTC). These materials can be obtained by high-temperature pyrolysis of organic precursors such as ethylene or furfuryl alcohol inside the zeolite pores, followed by HF dissolution of the zeolite template. ${ }^{34}$ The resulting ZTCs are three-dimensional carbon networks displaying varying degrees of order. More generally, ZTCs are porous particulate materials that consist of curved graphene moieties intergrown inside the pores with morphology of particles usually similar to the parent zeolite crystals. ZTCs are promising for numerous applications in catalysis, adsorption, optics, and fuel cells. ${ }^{35}$ It should also be mentioned that ZTC materials are known to possess dangling bonds, protected by the zeolite framework. ${ }^{36}$ This fact can explain the radical nature of the polyaromatic species confined in ZSM-5, as reported in our previous work. ${ }^{27}$

We modeled the MFI-templated carbon by filling a silicalite1 model with $\mathrm{sp}^{2}$-hybridized $\mathrm{C}$ atoms, placing acenes in the straight channels, connected by phenacene chains via the sinusoidal channels, and pyrene-like moieties in the intersections. ${ }^{27}$ The phenacene chains were found to offer the best balance between filling of the sinusoidal channels, the fit and flexibility as compared to other candidate polyaromatics. As a result linear acenes of infinite length lay along the straight channels in the b-direction and slightly twisted picene moieties connect the straight acenes to form a three-dimensional framework (Figure 13). Two types of micropores were found

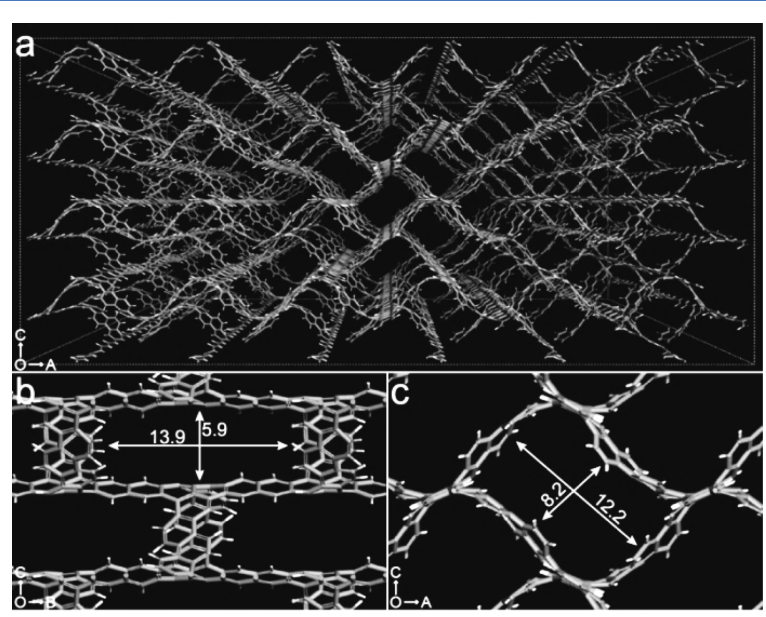

Figure 13. Structural model of an MFI-templated ZTC material: (a) view along $b$-axis in the direction of straight channels, (b) close view along the $a$-axis, and (c) close view along the $b$-axis.

in this model. The first type of pores runs along the $b$-direction and has dimensions of $8.2 \times 12.2 \AA$. The second type of pores can be viewed along the $a$-axis and has dimensions of $5.9 \times$ 13.9 A. These sizes are present in the NLDFT-derived pore size distribution of the liberated carbon material (Figure 12), which confirms the plausibility of the model.

The used model, however, does not account for the structural defects, such as missing linkages and incomplete intergrowth. An important aspect of ZTCs is that their crystallinity depends on the topology of the zeolite template. Highly crystalline ZTC materials with low defect densities and surface areas above $4000 \mathrm{~m}^{2} / \mathrm{g}$ can be obtained with threedimensional 12-membered ring (MR) zeolites, such as FAU,
BEA, and EMT. ${ }^{37}$ Synthesis of ZTC from the 10-MR MFI zeolite results in more disordered materials with no apparent crystalline structure, attributed to steric constraints. ${ }^{38}$ Such disorder was also observed in this work, manifested by a high mesoporous volume and moderate surface area. Nevertheless, the NMR, TG, electron microscopy, and Ar adsorption results obtained in this work are with the range of properties previously reported for MFI-templated ZTCs. ${ }^{39-41}$ We expect that the method of direct ZTC synthesis from methane over Mo/zeolite materials can be extended to other zeolite topologies as well. These results show that it is possible to synthesize microporous zeolite-templated carbons directly from methane, which will lead to new possibilities for these interesting materials.

\section{CONCLUSIONS}

Formation of heavy carbonaceous deposits is an intrinsic property of the MDA reaction. It is highly undesirable as large polyaromatic species block the zeolite pores and lead to the catalyst deactivation. However, these species also play an active intermediate role, as we showed by pulse reaction experiments and isotope labeling. Furthermore, within the sensitivity limit of ${ }^{13} \mathrm{C}$ NMR, the structure of coke, formed during MDA, does not depend on time on stream and acidity of the zeolite support. The often reported distinction between "soft" and "hard" coke can be explained solely by the $\mathrm{MoO}_{x}$-catalyzed combustion of near-surface carbon ("soft" coke feature) and diffusion limitations experienced by molecules of oxygen and combustion products when the zeolite micropores become gradually blocked by the polyaromatic molecules ("hard" coke feature). Finally, the carbon material formed during the MDA after dissolution of the zeolite framework possesses all properties of MFI-templated carbon materials. Conversion of methane over Mo/zeolite catalysts, therefore, offers a direct synthesis route from methane to zeolite-templated carbons.

\section{ASSOCIATED CONTENT}

S Supporting Information

The Supporting Information is available free of charge on the ACS Publications website at DOI: 10.1021/acscatal.8b02491.

Figures $\mathrm{S} 1-\mathrm{S} 3$ (PDF)

\section{AUTHOR INFORMATION}

\section{Corresponding Authors}

*Tel: +31-40-2478156. E-mail: n.a.kosinov@tue.nl.

*Tel: +31-40-2475178. E-mail: e.j.m.hensen@tue.nl.

ORCID $\odot$

Nikolay Kosinov: 0000-0001-8520-4886

Emiel J. M. Hensen: 0000-0002-9754-2417

Notes

The authors declare no competing financial interest.

\section{ACKNOWLEDGMENTS}

Financial support from the Sabic-NWO CATC1CHEM CHIPP project is gratefully acknowledged.

\section{REFERENCES}

(1) Schwach, P.; Pan, X.; Bao, X. Direct Conversion of Methane to Value-Added Chemicals over Heterogeneous Catalysts: Challenges and Prospects. Chem. Rev. 2017, 117, 8497-8520. 
(2) Kosinov, N.; Hensen, E. J. M. Nonoxidative Dehydroaromatization of Methane. Nanotechnology in Catalysis; Wiley-VCH: Weinheim, 2017; pp 469-482.

(3) Ismagilov, Z. R.; Matus, E. V.; Tsikoza, L. T. Direct Conversion of Methane on Mo/ZSM-5 Catalysts to Produce Benzene and Hydrogen: Achievements and Perspectives. Energy Environ. Sci. 2008, $1,526$.

(4) Cao, Z.; Jiang, H.; Luo, H.; Baumann, S.; Meulenberg, W. A.; Assmann, J.; Mleczko, L.; Liu, Y.; Caro, J. Natural Gas to Fuels and Chemicals: Improved Methane Aromatization in an OxygenPermeable Membrane Reactor. Angew. Chem., Int. Ed. 2013, 52, 13794-13797.

(5) Xue, J.; Chen, Y.; Wei, Y.; Feldhoff, A.; Wang, H.; Caro, J. Gas to Liquids: Natural Gas Conversion to Aromatic Fuels and Chemicals in a Hydrogen-Permeable Ceramic Hollow Fiber Membrane Reactor. ACS Catal. 2016, 6, 2448-2451.

(6) Morejudo, S. H.; Zanon, R.; Escolastico, S.; Yuste-Tirados, I.; Malerod-Fjeld, H.; Vestre, P. K.; Coors, W. G.; Martinez, A.; Norby, T.; Serra, J. M.; Kjølseth, C. Direct Conversion of Methane to Aromatics in a Catalytic Co-Ionic Membrane Reactor. Science 2016, $353,563-566$.

(7) Kosinov, N.; Coumans, F. J. A. G.; Uslamin, E.; Kapteijn, F.; Hensen, E. J. M. Selective Coke Combustion by Oxygen Pulsing During Mo/ZSM-5-Catalyzed Methane Dehydroaromatization. Angew. Chem., Int. Ed. 2016, 55, 15086-15090.

(8) Xu, Y.; Ma, H.; Yamamoto, Y.; Suzuki, Y.; Zhang, Z. Comparison of the Activities of Binder-Added and Binder-Free Mo/HZSM-5 Catalysts in Methane Dehydroaromatization at $1073 \mathrm{~K}$ in Periodic $\mathrm{CH}_{4}-\mathrm{H}_{2}$ Switch Operation Mode. J. Nat. Gas Chem. 2012, 21, 729744.

(9) Xu, Y.; Song, Y.; Zhang, Z. G. A Binder-Free Fluidizable Mo/ HZSM-5 Catalyst for Non-Oxidative Methane Dehydroaromatization in a Dual Circulating Fluidized Bed Reactor System. Catal. Today 2017, 279, 115-123.

(10) Portilla, M. T.; Llopis, F. J.; Martínez, C. Non-Oxidative Dehydroaromatization of Methane: An Effective Reaction-regeneration Cyclic Operation for Catalyst Life Extension. Catal. Sci. Technol. 2015, 5, 3806-3821.

(11) Kosinov, N.; Coumans, F. J. A. G.; Li, G.; Uslamin, E.; Mezari, B.; Wijpkema, A. S. G.; Pidko, E. A.; Hensen, E. J. M. Stable Mo/ HZSM-5 Methane Dehydroaromatization Catalysts Optimized for High-Temperature Calcination-Regeneration. J. Catal. 2017, 346, 125-133.

(12) Bauer, F.; Karge, H. G. Characterization of Coke on Zeolites. Mol. Sieves 2006, 5, 249-364.

(13) Ma, D.; Wang, D.; Su, L.; Shu, Y.; Xu, Y.; Bao, X. Carbonaceous Deposition on Mo/HMCM-22 Catalysts for Methane Aromatization: A TP Technique Investigation. J. Catal. 2002, 208, 260-269.

(14) Liu, H.; Li, Y.; Shen, W.; Bao, X.; Xu, Y. Methane Dehydroaromatization over Mo/HZSM-5 Catalysts in the Absence of Oxygen: Effects of Silanation in HZSM-5 Zeolite. Catal. Today 2004, 95, 65-73.

(15) Liu, H.; Bao, X.; Xu, Y. Methane Dehydroaromatization under Nonoxidative Conditions over Mo/HZSM-5 Catalysts: Identification and Preparation of the Mo Active Species. J. Catal. 2006, 239, 441450.

(16) Li, Y.; Liu, L.; Huang, X.; Liu, X.; Shen, W.; Xu, Y.; Bao, X. Enhanced Performance of Methane Dehydro-Aromatization on MoBased HZSM-5 Zeolite Pretreated by $\mathrm{NH}_{4}$ F. Catal. Commun. 2007, 8, $1567-1572$.

(17) Liu, B. S.; Jiang, L.; Sun, H.; Au, C. T. XPS, XAES, and TG/ DTA Characterization of Deposited Carbon in Methane Dehydroaromatization over Ga-Mo/ZSM-5 Catalyst. Appl. Surf. Sci. 2007, 253, $5092-5100$.

(18) Tessonnier, J.-P.; Louis, B.; Rigolet, S.; Ledoux, M. J.; PhamHuu, C. Methane Dehydro-Aromatization on Mo/ZSM-5: About the Hidden Role of Brønsted Acid Sites. Appl. Catal., A 2008, 336, 7988.
(19) Masiero, S. S.; Marcilio, N. R.; Perez-Lopez, O. W. Aromatization of Methane over Mo-Fe/ZSM-5 Catalysts. Catal. Lett. 2009, 131, 194-202.

(20) Song, Y.; Xu, Y.; Suzuki, Y.; Nakagome, H.; Zhang, Z. G. A Clue to Exploration of the Pathway of Coke Formation on Mo/ HZSM-5 Catalyst in the Non-Oxidative Methane Dehydroaromatization at $1073 \mathrm{~K}$. Appl. Catal., A 2014, 482, 387-396.

(21) Song, Y.; Xu, Y.; Suzuki, Y.; Nakagome, H.; Ma, X.; Zhang, Z. G. The Distribution of Coke Formed over a Multilayer Mo/HZSM-5 Fixed Bed in $\mathrm{H}_{2}$ Co-Fed Methane Aromatization at $1073 \mathrm{~K}$ : Exploration of the Coking Pathway. J. Catal. 2015, 330, 261-272.

(22) Tempelman, C. H. L.; Zhu, X.; Hensen, E. J. M. Activation of Mo/HZSM-5 for Methane Aromatization. Chin. J. Catal. 2015, 36, 829-837.

(23) Tempelman, C. H. L.; De Rodrigues, V. O.; Van Eck, E. R. H.; Magusin, P. C. M. M.; Hensen, E. J. M. Desilication and Silylation of Mo/HZSM-5 for Methane Dehydroaromatization. Microporous Mesoporous Mater. 2015, 203, 259-273.

(24) Song, Y.; Zhang, Q.; Xu, Y.; Zhang, Y.; Matsuoka, K.; Zhang, Z. G. Coke Accumulation and Deactivation Behavior of MicrozeoliteBased Mo/HZSM-5 in the Non-Oxidative Methane Aromatization under Cyclic $\mathrm{CH}_{4}-\mathrm{H}_{2}$ feed Switch Mode. Appl. Catal., A 2017, 530, $12-20$.

(25) Weckhuysen, B. M.; Rosynek, M. P.; Lunsford, J. H. Characterization of Surface Carbon Formed during the Conversion of Methane to Benzene over Mo/H-ZSM-5 Catalysts. Catal. Lett. 1998, 52, 31-36.

(26) Tempelman, C. H. L.; Hensen, E. J. M. On the Deactivation of Mo/HZSM-5 in the Methane Dehydroaromatization Reaction. Appl. Catal., B 2015, 176-177, 731-739.

(27) Kosinov, N.; Wijpkema, A. S. G.; Uslamin, E.; Rohling, R.; Coumans, F. J. A. G.; Mezari, B.; Parastaev, A.; Poryvaev, A. S.; Fedin, M. V.; Pidko, E. A.; Hensen, E. J. M. Confined Carbon Mediating Dehydroaromatization of Methane over Mo/ZSM-5. Angew. Chem., Int. Ed. 2018, 57, 1016-1020.

(28) Olsbye, U.; Svelle, S.; Bjørgen, M.; Beato, P.; Janssens, T. V. W.; Joensen, F.; Bordiga, S.; Lillerud, K. P. Conversion of Methanol to Hydrocarbons: How Zeolite Cavity and Pore Size Controls Product Selectivity. Angew. Chem., Int. Ed. 2012, 51, 5810-5831.

(29) Kosinov, N.; Sripathi, V. G. P.; Hensen, E. J. M. Microporous Mesoporous Mater. 2014, 194, 24-30.

(30) Okkel, L. G.; Gavrilov, V. Y.; Fenelonov, V. B.; Echevskii, G. V. Studies of Coke Distribution in High-Silica ZSM Zeolites. React. Kinet. Catal. Lett. 1986, 30, 269-274.

(31) Milanesio, M.; Artioli, G.; Gualtieri, A. F.; Palin, L.; Lamberti, C. Template Burning inside TS- 1 and Fe-MFI Molecular Sieves: An in Situ XRPD Study. J. Am. Chem. Soc. 2003, 125, 14549-14558.

(32) Kosinov, N.; Coumans, F. J. A. G.; Uslamin, E. A.; Wijpkema, A. S. G.; Mezari, B.; Hensen, E. J. M. Methane Dehydroaromatization by Mo/HZSM-5: Mono- or Bifunctional Catalysis? ACS Catal. 2017, 7, 520-529.

(33) Solum, M. S.; Sarofim, A. F.; Pugmire, R. J.; Fletcher, T. H.; Zhang, H. 13C NMR Analysis of Soot Produced from Model Compounds and a Coal. Energy Fuels 2001, 15, 961-971.

(34) Kim, K.; Lee, T.; Kwon, Y.; Seo, Y.; Song, J.; Park, J. K.; Lee, H.; Park, J. Y.; Ihee, H.; Cho, S. J.; Ryoo, R. Lanthanum-Catalysed Synthesis of Microporous 3D Graphene-like Carbons in a Zeolite Template. Nature 2016, 535, 131-135.

(35) Nishihara, H.; Kyotani, T. Zeolite-Templated Carbons Three-Dimensional Microporous Graphene Frameworks. Chem. Commun. 2018, 54, 5648-5673.

(36) Nishihara, H.; Imai, K.; Itoi, H.; Nomura, K.; Takai, K.; Kyotani, T. Formation Mechanism of Zeolite-Templated Carbons. Tanso 2017, 2017, 169-174.

(37) Nishihara, H.; Kyotani, T. Templated Nanocarbons for Energy Storage. Adv. Mater. 2012, 24, 4473-4498.

(38) Meyers, C. J.; Shah, S. D.; Patel, S. C.; Sneeringer, R. M.; Bessel, C. A.; Dollahon, N. R.; Leising, R. A.; Takeuchi, E. S. Tesmplated Synthesis of Carbon Materials from Zeolites (Y, Beta, and 
ZSM-5) and a Montmorillonite Clay (K10): Physical and Electrochemical Characterization. J. Phys. Chem. B 2001, 105, 2143-2152.

(39) Kyotani, T.; Ma, Z.; Tomita, A. Template Synthesis of Novel Porous Carbons Using Various Types of Zeolites. Carbon 2003, 41, $1451-1459$.

(40) Liu, L.; Ma, D.; Zheng, H.; Li, X.; Cheng, M.; Bao, X. Synthesis and Characterization of Microporous Carbon Nitride. Microporous Mesoporous Mater. 2008, 110, 216-222.

(41) Valero-Romero, M. J.; Márquez-Franco, E. M.; Bedia, J.; Rodríguez-Mirasol, J.; Cordero, T. Hierarchical Porous Carbons by Liquid Phase Impregnation of Zeolite Templates with Lignin Solution. Microporous Mesoporous Mater. 2014, 196, 68-78. 